\title{
Janus粒子的表面改性及其对共混聚合物的 增容性能研究
}

\author{
梁新铖, 张操, 贺爱华, 聂华荣* \\ 山东省烯烃催化与聚合重点实验室, 橡塑材料与工程教育部重点实验室, 青岛科技大学高分子科学与工程学院, 青岛 266042 \\ *通讯作者, E-mail: niehr@iccas.ac.cn
}

收稿日期：2018-01-05; 接受日期：2018-03-12; 网络版发表日期：2018-04-25

国家自然科学基金(编号：51773105，51473083)、山东省自然科学基金(编号：ZR2016EMM05)、国家重点基础研究发展计划(编号： 2015CB654700, 2015CB354706)、山东省重大基础研究项目(编号: ZR2017ZA0304)和泰山学者工程项目资助

\begin{abstract}
摘要本文以雪人状的 $\mathrm{SiO}_{2} @ \mathrm{PDVB}$ Janus粒子为模板, 通过表面改性获得具有不同表面性质的Janus粒子, 包括 在 $\mathrm{SiO}_{2}$ 半球分别引入聚丁二烯 $(\mathrm{PBd})$ 与聚异戊二烯 $(\mathrm{PI})$, 以增加 $\mathrm{SiO}_{2} @ \mathrm{PDVB}$ Janus粒子中无机的 $\mathrm{SiO}_{2}$ 半球对聚合物 基质的亲和性. 随后将改性前后的Janus粒子分散到聚苯乙烯(PS)/顺丁橡胶(PBd)及聚苯乙烯(PS)/异戊橡胶(PI)的 共混体系中, 考察了不同Janus粒子对共混物相结构的影响. 结果发现, 与未改性的Janus粒子相比，引入聚合物刷 的Janus粒子可更有效地提高其界面增容效果, 抑制共混物相分离的动力学.
\end{abstract}

关键词 Janus粒子, 表面改性, 增容, 聚合物共混物

\section{1 引言}

Janus粒子是一类表面同时具有两种不同化学组 成并严格分区的新型颗粒 ${ }^{[14]}$, 在固体乳化剂、界面增 容、界面催化、功能涂层、细胞诊断与治疗等应用领 域取得了重要的研究进展 ${ }^{[5]}$. 作为界面增容剂, Janus 粒子独特的结构特征使其兼具表面活性剂的双亲性和 固体粒子的Pickering效应，比均质纳米粒子或嵌段共 聚物具有更强的稳定界面作用，在聚合物共混物中 表现出优异的增容性，有望成为新一代理想的增容 剂 $^{[10]}$.

中国科学院化学研究所郭红霞研究员课题组 ${ }^{[11,12]}$ 模拟了Janus粒子对不相容共混聚合物相分离行为的
影响, 结果表明, 与均质粒子相比, Janus粒子可以更有 效地抑制相区的增长, 降低相区的尺寸. 实验研究亦表 明 ${ }^{[13]}$ ，在Janus粒子的不同表面区间，分别引入与共混 聚合物化学性质相匹配的聚合物刷, 可显著改善Janus 粒子在聚合物相界面的聚集行为. 此外, 聚合物刷与共 混聚合物分子的相互扩散，还可抑制因体系构象熵损 失引起的表面能变化, 增强粒子在界面的稳定性. Müller等 ${ }^{[14,15]}$ 制备了带聚苯乙烯(PS)和聚甲基丙烯酸 甲酯(PMMA)分子刷的Janus粒子, 观察其对PS/PMMA 共混体系的增容效果, 发现即使在高温和高剪切作用 下，粒子仍能稳定地聚集在界面，表现出优异的界面 活性和增容性. 杭州师范大学李勇进教授课题组 ${ }^{[16,17]}$ 利用嵌段共聚物原位聚合方法，制备了带聚甲基丙烯

引用格式: Liang X, Zhang C, He A, Nie H. Surface modification of Janus particles and their compatibilization in polymer blends. Sci Sin Chim, 2018, 48: 527-534, doi: $10.1360 / \mathrm{N} 032018-00005$ 
酸甲酯(PMMA)和聚乳酸(PLLA)分子刷的Janus纳米 胶束，同样发现，其对聚偏氟乙烯(PVDF)和聚乳酸 (PLLA)共混体系具有良好的增容效果.

尽管Janus粒子对相结构的调控机制目前还不是 非常清楚，但一致认为Janus粒子表面的双亲性可有效 降低体系表面能, 是决定其界面活性的关键因素. 本文 以雪人状的 $\mathrm{SiO}_{2} @ \mathrm{PDVB}$ Janus粒子为模板 ${ }^{[18,19]}$, 通过 表面改性的方法在 $\mathrm{SiO}_{2}$ 半球分别引入聚丁二烯 $(\mathrm{PBd})$ 及聚异戊二烯(PI)的分子链，以平衡Janus粒子两半球 对高分子基体的亲和性，达到对聚合物共混物更好的 增容效果.

\section{2 实验部分}

\section{1 主要原料}

丁二烯, 聚合级, 齐鲁石化(中国); 异戊二烯, 聚合 级, 山东华聚高分子材料有限公司 (中国); $\mathrm{SiO}_{2}$ @ PDVB Janus微球，东营雅努斯新材料科技有限公司 (中国); 正丁基锂, $2.5 \mathrm{~mol} / \mathrm{L}$, 分散于环己烷，阿拉丁 试剂(中国); 氯乙基三甲氧基硅烷，99\%，阿拉丁试剂; 三乙胺, $99 \%$, 阿拉丁试剂; 聚苯乙烯, $M \mathrm{w}=270 \mathrm{k}$, 阿拉 丁试剂; 异戊橡胶 $(\mathrm{PI}), M_{\mathrm{W}}=610 \mathrm{k}$; 顺丁橡胶BR9000 $(\mathrm{PBd}), M \mathrm{w}=500 \mathrm{k}$, 购自青岛伊科思新企业(中国); 环己 烷，分析纯，天津博迪化工股份有限公司(中国)，使用 前需进行精制，具体为将适量的钠与二苯甲酮加入到 环己烷中加热回流, 待溶液变为深蓝色, 将环己烷蒸 馏，收集备用. 二氯甲烷，分析纯，天津市富宇精细化 工有限公司(中国); 甲苯，分析纯，莱阳经济开发区精 细化工厂(中国).

\section{2 实验步骤}

\subsection{1雪人状 $\mathrm{SiO}_{2} @$ PDVB Janus粒子的表面改性}

如图1所示，采用接枝到的方法将聚丁二烯 $(\mathrm{PBd})$ 或聚异戊二烯(PI)分子链接枝到无机 $\mathrm{SiO}_{2}$ 半球表面, 以 提高Janus粒子与聚合物基底的相容性. 阴离子聚合制 备的带有活性中心的PBd分子链与硅烷偶联剂上的卤 素原子反应, 获得大分子的硅烷偶联剂(I). 随后, 在三 乙胺的催化下，大分子偶联剂中的甲氧基与 $\mathrm{SiO}_{2} @$ PDVB Janus粒子中 $\mathrm{SiO}_{2}$ 半球上的羟基反应， $\mathrm{PBd}$ 分子 链即被接枝到二氧化硅半球(II), 形成单侧接枝的聚合 物分子刷, 提高 $\mathrm{SiO}_{2}$ 半球与有机高分子基底的亲和性,
平衡两半球与聚合物基质的界面张力. $\mathrm{PI}$ 改性 $\mathrm{SiO}_{2} @$ PDVB Janus粒子与PBd改性方法一致，只需将PBd换 成PI即可.

\subsection{2 丁二烯的阴离子聚合}

将 $100 \mathrm{~mL}$ 洁净的聚合瓶加热烘烤并抽排, 用氮气 置换3次后, 在聚合瓶内保持氮气氛围. 随后, 向聚合瓶 中加入 $30 \mathrm{~mL}$ 精制的环己烷，并采用气相液化法加入 $6 \mathrm{~g} 丁$ 二烯单体，以设定浓度 $[\mathrm{M}] /[\mathrm{I}]=1000$ 加入正丁基 锂引发剂. 设置反应温度为 $25^{\circ} \mathrm{C}$, 反应时间为 $5 \mathrm{~h}$. 反 应结束后保持装置密闭体系不变，准备进行下一步的 反应。

\subsection{3 大分子硅烷偶联剂的制备}

向2.2.2 小节中完成制备的带有活性中心的聚丁二 烯环己烷溶液中, 加入 $2 \mathrm{~mL}$ 氯乙基三甲氧基硅烷，反 应温度为 $25^{\circ} \mathrm{C}$, 反应时间为 $12 \mathrm{~h}$, 在聚丁二烯分子链的 末端引入硅烷偶联剂. 所得产物旋转蒸发后, 在 $45^{\circ} \mathrm{C}$ 真 空干燥箱中干燥至恒重, 准备下一步反应.

\subsubsection{PBd-SiO ${ }_{2} @ P D V B$ 的合成}

将0.5 $\mathrm{g}$ 的 $\mathrm{SiO}_{2} @ \mathrm{PDVB}$ Janus粒子分散在甲苯溶液 中, 加入 2.2 .3 小节中制备的 $1 \mathrm{~g}$ 大分子硅烷偶联剂与 0.5 $\mathrm{mL}$ 的三乙胺溶液, 反应温度为 $25^{\circ} \mathrm{C}$, 反应时间为 $24 \mathrm{~h}$, 用二氯甲烷清洗粒子，直至上清液中检测不出聚丁二 烯分子链. 大分子偶联剂末端的甲氧基与 $\mathrm{SiO}_{2} @ \mathrm{PDVB}$ Janus粒子中 $\mathrm{SiO}_{2}$ 半球表面的羟基反应，将聚丁二烯分 子链接枝到二氧化硅一侧, 获得聚合物刷改性的Janus 粒子(PBd-SiO $\left.\mathrm{P}_{2} @ \mathrm{PDVB}\right)$, 可观察到粒子呈黏性状态. $\mathrm{PI}-\mathrm{SiO}_{2} @ \mathrm{PDVB}$ 与 $\mathrm{PBd}-\mathrm{SiO}_{2} @ \mathrm{PDVB}$ Janus粒子的制备 方法相同, 只需将丁二烯换成异戊二烯, 聚丁二烯变成 聚异戊二烯.

\subsection{5 共混物样品的制备}

将改性前后的Janus粒子分别超声分散于 $5 \mathrm{~mL}$ 二 氯甲烷中, 随后将 $60 \mathrm{mg}$ 聚苯乙烯与 $40 \mathrm{mg}$ 顺丁橡胶或 $60 \mathrm{mg}$ 聚苯乙烯与 $40 \mathrm{mg}$ 异戊橡胶分别溶解于含Janus 粒子的二氯甲烷中，加入 $0.3 \%$ 的防老剂 264 配制成浓 度为 $2 \%$ 的聚合物溶液. 上述聚合物溶液在 (SC100-SS, BestTools，LLC，美国)匀胶机室温旋涂(3000 r/min, 60 s)成膜, 样品置于室温下干燥 $1 \mathrm{~h}$. 


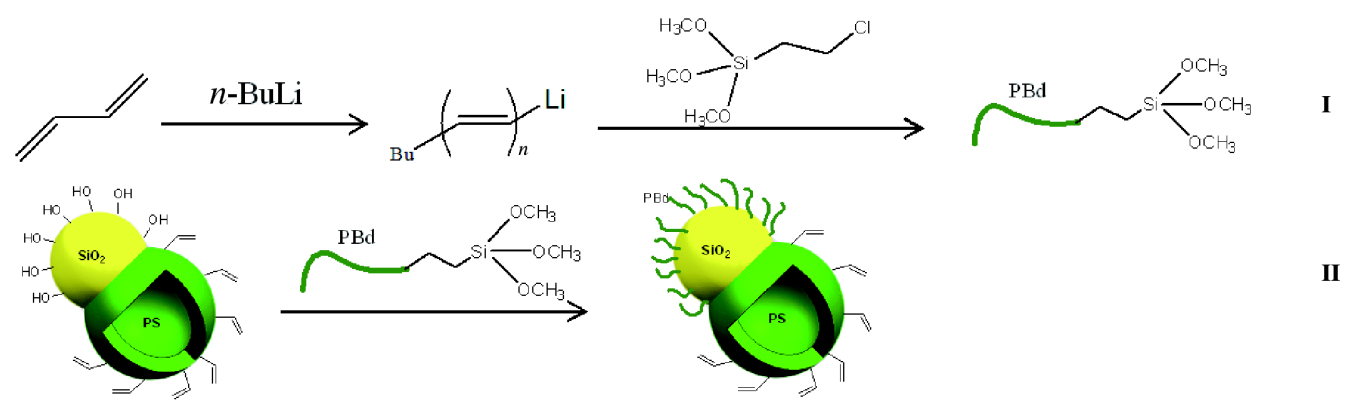

图 1 二氧化硅一侧接枝聚丁二烯制备PBd- $\mathrm{SiO}_{2} @ \mathrm{PDVB}$ 像胶杂化Janus粒子示意图(网络版彩图)

Figure 1 Illustrative synthesis of the Janus rubber hybrid particles ( $\left.\mathrm{PBd}-\mathrm{SiO}_{2} @ \mathrm{PDVB}\right)$ by successively grafting polybutadiene chains onto $\mathrm{SiO}_{2}$ side (color online).

\section{3 测试表征}

采用Bruker (500MHZ, 德国)核磁共振分析仪分析 聚合物刷的微观结构, 样品在常温下用気代氯仿(含 $1 \% \mathrm{TMS}$ )溶解, 配制成 $2 w / v \%$ 的溶液. 采用JEM 2100 的透射电镜(TEM, 日本)观察Janus粒子的形貌, 工作电 压为 $200 \mathrm{kV}$. 共混物的相形貌采用奥林巴斯BX51相差 显微镜(日本)观察. 共混物膜置于 $150^{\circ} \mathrm{C}$ 的热台上退火 处理不同时间后观察相形貌. 分子量及分布采用HLC8320 凝胶渗透色谱仪测试, 以四氢呋喃为流动相, 以单 分散的聚苯乙烯为标样. 柱温为 $30^{\circ} \mathrm{C}$, 示差检温器为 $35^{\circ} \mathrm{C}$, 流动相流速为 $1.0 \mathrm{~mL} / \mathrm{min}$, 进样量为 $50 \mu \mathrm{L}$. 采用 型号为TG209F1Libra (Netzsch, 德国)的热失重仪分析 聚合物的接枝密度, 以 $10{ }^{\circ} \mathrm{C} / \mathrm{min}$ 的升温速率将样品从 室温升至 $900^{\circ} \mathrm{C}$, 接枝密度计算公式 ${ }^{[20]}$ 如下:

$$
G_{r}=\frac{W_{1}}{1-W_{1}}-\frac{W_{2}}{1-W_{2}},
$$

其中, $G_{r}$ 代表重量接枝密度, $W_{1}$ 代表未接枝Janus粒子 在热失重仪中升温到 $900^{\circ} \mathrm{C}$ 后的残余无机物含量, $W_{2}$ 代表接枝Janus粒子在热失重仪中升温到 $900^{\circ} \mathrm{C}$ 后的残 余无机物含量.

\section{3 结果与讨论}

\section{1 硅烷偶联剂接枝PBd的氢谱分析}

图2为硅烷偶联剂接枝聚丁二烯分子链的 ${ }^{1} \mathrm{H} N \mathrm{NR}$ 谱图. 图中代表PBd分子链顺式1,4-结构双键上氢的 $\mathrm{a} 1$ 峰非常明显, 代表1,2-结构的侧链上双键上氢的a2较 弱, a3 归属为甲氧基- $\mathrm{OCH} 3$ 氢原子的化学位移, 甲氧 基氢原子的化学位移的存在证明硅烷偶联剂接枝上聚

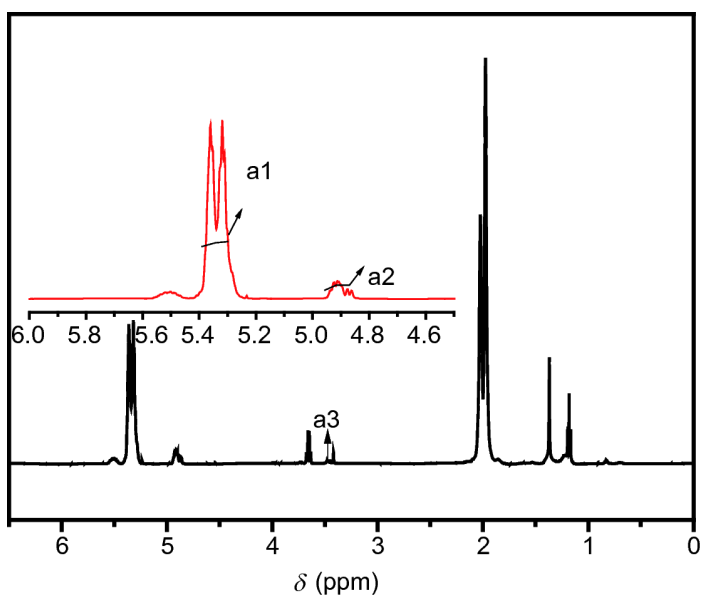

图 2 硅烷偶联剂接枝聚丁二烯分子链的 ${ }^{1} \mathrm{H}-\mathrm{NMR}$ (网络版 彩图)

Figure $2{ }^{1} \mathrm{H}$ NMR of silane coupling agent grafting PBd (color online).

丁二烯分子链. 经计算, 聚丁二烯分子链为高顺式1,4结构, 其1,4-结构占93 $\mathrm{mol} \%, 1,2-$ 结构占7 $\mathrm{mol} \%{ }^{[21]}$.

\section{2 $\mathrm{PI}-\mathrm{SiO}_{2} @ \mathrm{PDVB}$ 的热失重研究}

图3给出了改性前后两种粒子的热失重曲线, 由于 聚合物刷的引入，改性后无机 $\mathrm{SiO}_{2}$ 的固体含量明显低 于改性前的Janus粒子. 经计算, $\mathrm{PI}-\mathrm{SiO}_{2} @ \mathrm{PDVB}$ 的 质量接枝密度为 $47.8 \mathrm{wt} \%$. 另经测试, 接枝 $\mathrm{PBd} 、 \mathrm{PI}$ 分 子刷的分子量分别为 $64 \mathrm{k} 、 114 \mathrm{k}$, 分散系数分别为 $1.4 、 1.3$.

\section{3 $\mathrm{SiO}_{2} @ P D V B$ 改性前后的形貌}

图4(a)为DMF刻蚀PS后的 $\mathrm{SiO}_{2} @ \mathrm{PDVB}$ Janus粒子 的形貌. $\mathrm{SiO}_{2} @$ PDVB Janus粒子呈雪人状结构, 大头 


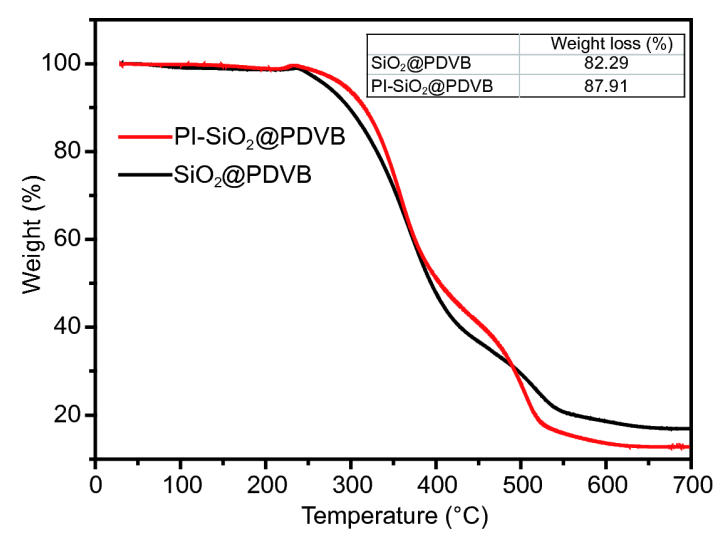

图 $3 \mathrm{SiO}_{2} @ \mathrm{PDVB}$ 与 $\mathrm{PI}-\mathrm{SiO}_{2} @ \mathrm{PDVB}$ 粒子的热失重曲线(网 络版彩图)

Figure 3 TGA curves of $\mathrm{SiO}_{2} @$ PDVB and PI-SiO $@$ PDVB (color online).

PDVB一侧为中空结构, 并呈现介孔结构, 小头的二氧 化硅呈光滑状态. $\mathrm{PDVB}$ 半球在 $N, N$-二甲基甲酰胺 $(\mathrm{DMF})$ 刻蚀前为PDVB-PS复合物, DMF刻蚀掉线性的 PS分子链后，在交联的PDVB半球留下介孔结构。图 4(b)为 $\mathrm{SiO}_{2}$ 半球接枝 $\mathrm{PBd}$ 后 $\mathrm{SiO}_{2} @ \mathrm{PDVB} \mathrm{Janus}$ 粒子形 貌. 当 $\mathrm{PBd}$ 分子链接枝在二氧化硅半球时, 原本光滑的 表面可观察到明显的聚合物层, 随着溶剂的挥发, 接枝 聚合物分子刷的一侧相互靠拢，自组装形成超结构(13), 两侧异性的Janus粒子之所以具有两亲性, 自组装是 其中一项重要的因素 ${ }^{[22,23]}$.

\subsection{PBd-SiO $@$ @PDVB增容PS/PBd共混体系的 研究}

$\mathrm{PS} / \mathrm{PBd}$ 为不相容共混体系, 在旋转涂膜的过程中, 由于溶剂的诱导作用 ${ }^{[24]}, \mathrm{PS} / \mathrm{PBd}$ 共混物呈现出明显的 两相结构(图5), 在添加不同含量的PBd- $\mathrm{SiO}_{2} @ \mathrm{PDVB}$ Janus粒子后，可以发现相结构随之发生变化，相区尺 寸略有变小, 同时高浓度粒子在 $\mathrm{PS} / \mathrm{PBd}$ 共混物中出现 了明显的聚集现象(图5(c,d)). 图6为 $\mathrm{PS} / \mathrm{PBd}$ 共混物在 $150^{\circ} \mathrm{C}$ 退火处理 2 h后, 不同浓度PBd- $\mathrm{SiO}_{2} @ \mathrm{PDVB} \mathrm{Ja}-$ nus粒子对相形貌的影响.

显然，与退火前相比，PS/PBd共混物的两相结构 更加明显，加入粒子后的 $\mathrm{PS} / \mathrm{PBd}$ 共混物相区尺寸明显 减小, 且粒子含量越高, 相区尺寸越小. 当粒子含量增 加到 $15 \%$ 时, PS 与 $\mathrm{PBd}$ 的双连续相结构出现, 但不可否 认，15 wt \% 高含量PBd- $\mathrm{SiO}_{2} @$ PDVB Janus粒子在PS/ $\mathrm{PBd}$ 共混物中同样聚集明显, 这是由于粒子的加入降 低了聚合物分子链的构象熵，当粒子浓度增大到一定 程度后，体系熵损失太大而不能使粒子稳定在聚合物 中, 粒子聚集变得明显, 并从聚合物相中分离出来.

图7为不添加或添加不同Janus粒子的PS/PBd共混 体系在 $150^{\circ} \mathrm{C}$ 退火 $2 \mathrm{~h}$ 后的相形貌. 显然, 固体粒子的加 入可有效调控共混聚合物的相结构. 与未修饰的 $\mathrm{SiO}_{2} @ \mathrm{PDVB}$ Janus粒子相比, 改性后的PBd-SiO ${ }_{2} @$ PDVB Janus固体粒子增容效果更明显. 这是由于改性
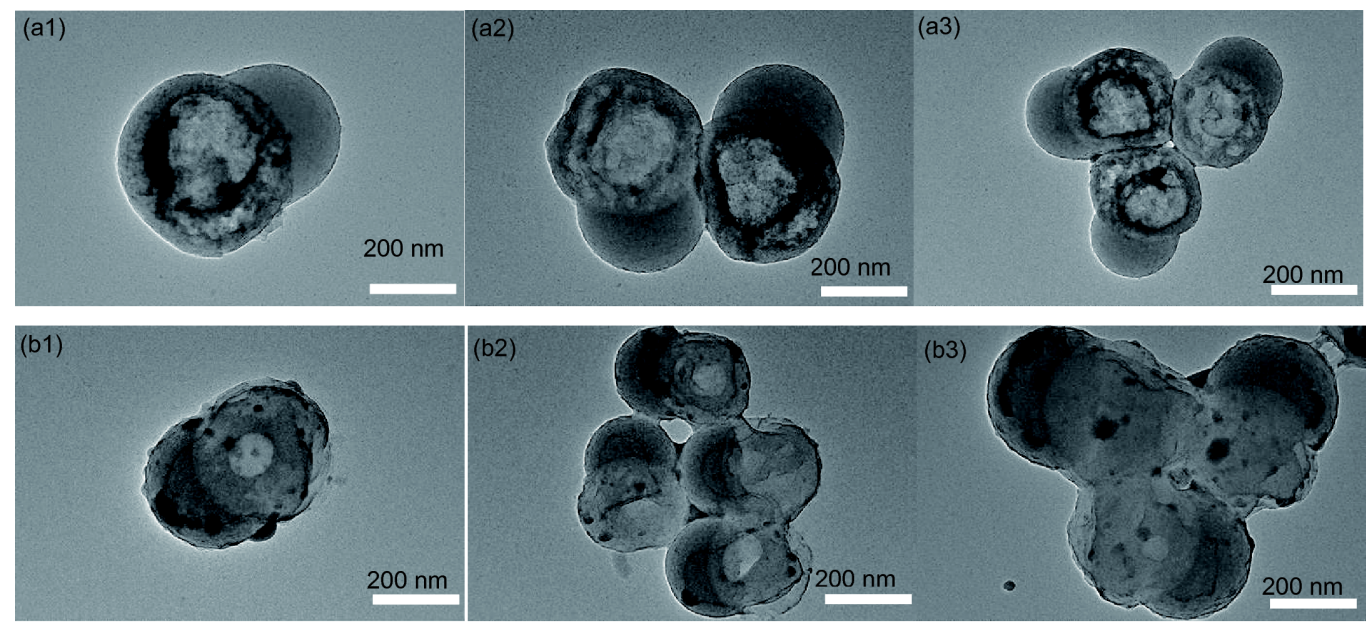

图 4 (a1 a3) 刻蚀线性聚苯乙烯模板后的 $\mathrm{SiO}_{2} @ \mathrm{PDVB}$ 粒子的透射电镜图; (b1 b3) PBd- $\mathrm{SiO}_{2} @ \mathrm{PDVB}$ 粒子经四氧化钉染色后 的透射电镜图

Figure 4 TEM images of $\mathrm{SiO}_{2} @$ PDVB particles after the etching of linear polystyrene (a1 a3) and $\mathrm{PBd}-\mathrm{SiO} @$ PDVB particles stained by ruthenium tetroxide (b1 b3). 

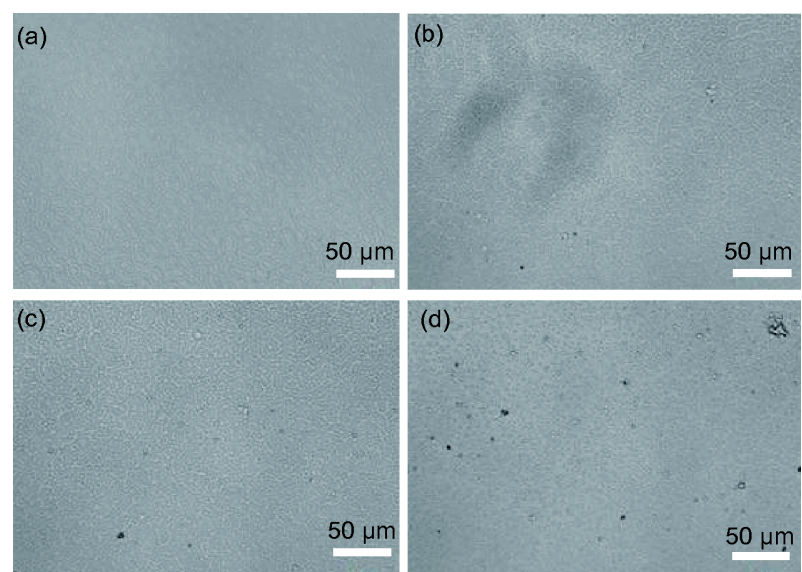

图 5 PS60PBd40与 $0 \mathrm{wt} \%$ (a), $5 \mathrm{wt} \%$ (b), $10 \mathrm{wt} \%$ (c), $15 \mathrm{wt} \%$ (d) $\mathrm{PBd}-\mathrm{SiO}_{2} @ \mathrm{PDVB}$ 粒子共混后的相形貌

Figure 5 Phase contrast optical microscopic images of the PS60PBd40 blends with the varied concentration of $\mathrm{PBd}-\mathrm{SiO}_{2} @ \mathrm{PDVB}$ (wt\%): (a) 0, (b) 5, (c) 10, (d) 15.

前, $\mathrm{SiO}_{2} @ \mathrm{PDVB}$ Janus粒子中的PDVB与PS良好的亲 和性，粒子更易倾向于在PS相分散，改性后，接枝在 $\mathrm{SiO}_{2}$ 半球的 $\mathrm{PBd}$ 分子链与共混聚合物基质 $\mathrm{PBd}$ 亲和性 好，从而在一定程度上平衡了Janus粒子对共混聚合物 两相的亲和性，提高了其界面活性及其对共混聚合物 相分离动力学的抑制, 如图7(c)所示, PS/PBd共混聚合 物具有更小的相尺寸.

\subsection{PI-SiO $@$ @PDVB增容PS/PI共混体系的研究}

采用相同的合成方法，将聚异戊二烯(PI)接枝到 $\mathrm{SiO}_{2} @ P D V B$ 粒子上，合成出带有PI分子链的固体粒 子, $\mathrm{PI}-\mathrm{SiO}_{2} @ \mathrm{PDVB}$. 将PI-SiO $\mathrm{Si}_{2} @ \mathrm{PDVB}$ Janus粒子添 加到PS/PI (6/4 wt $\% / \mathrm{wt} \%)$ 共混体系后, 旋转涂膜, 并于 $150^{\circ} \mathrm{C}$ 退火100 min, 观察PI- $\mathrm{SiO}_{2} @ \mathrm{PDVB}$ 对PS/PI共混 体系的增容作用.
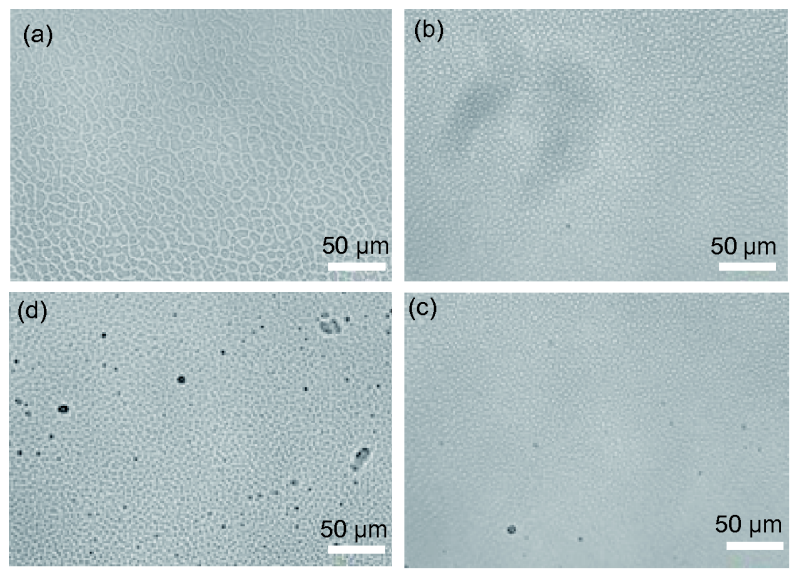

图 6 PS60PBd40与 $0 \mathrm{wt} \%$ (a), $5 \mathrm{wt} \%$ (b), $10 \mathrm{wt} \%$ (c), $15 \mathrm{wt} \%$ (d) $\mathrm{PBd}-\mathrm{SiO}_{2} @ \mathrm{PDVB}$ 粒子共混, 于 $150{ }^{\circ} \mathrm{C}$ 退火 $2 \mathrm{~h}$ 后的相形貌 Figure 6 Phase contrast optical microscopic images of the PS60PBd40 blends with the varied concentration of PBd-SiO $\mathrm{S}_{2} @$ PDVB (wt\%): (a) 0 , (b) 5, (c) 10 , (d) 15 annealed at $150^{\circ} \mathrm{C}$ for $2 \mathrm{~h}$.

图8为未修饰的 $\mathrm{SiO}_{2} @ \mathrm{PDVB}$ Janus粒子对PS/PI共 混物相形貌的影响. 可以看出, 低于 $5 \mathrm{wt} \%$ 含量的 $\mathrm{SiO}_{2} @$ PDVB Janus粒子对PS/PI增容作用并不明显, 高 含量的 $\mathrm{SiO}_{2} @$ PDVB Janus粒子在共混物中的分散变得 困难, 粒子的团聚现象变得严重. 这是由于未改性前, 纯固体粒子对 PS表现较强的亲和性, 界面活性不 强, 主要通过物理吸附联接两相, 因而增容效果不 佳 ${ }^{[25]}$.

接枝聚异戊二烯分子链的固体粒子PI- $\mathrm{SiO}_{2} @$ $\mathrm{PDVB}$, 填充到 $\mathrm{PS} / \mathrm{IR}$ 共混体系后, 在 $150^{\circ} \mathrm{C}$ 退火 $100 \mathrm{~min}$ 后的相形貌, 如图9所示. 可以看出, 改性后的固体粒子 填充到PS/PI共混体系中, 没有严重的团聚现象. PS/PI 共混物表现为明显的两相结构, 且随着粒子含量的增 加, 相结构的特征尺寸减少, 与未修饰的 $\mathrm{SiO}_{2} @ \mathrm{PDVB}$ Janus粒子不同, PI分子链在 $\mathrm{SiO}_{2}$ 半球的引入, 降低了共
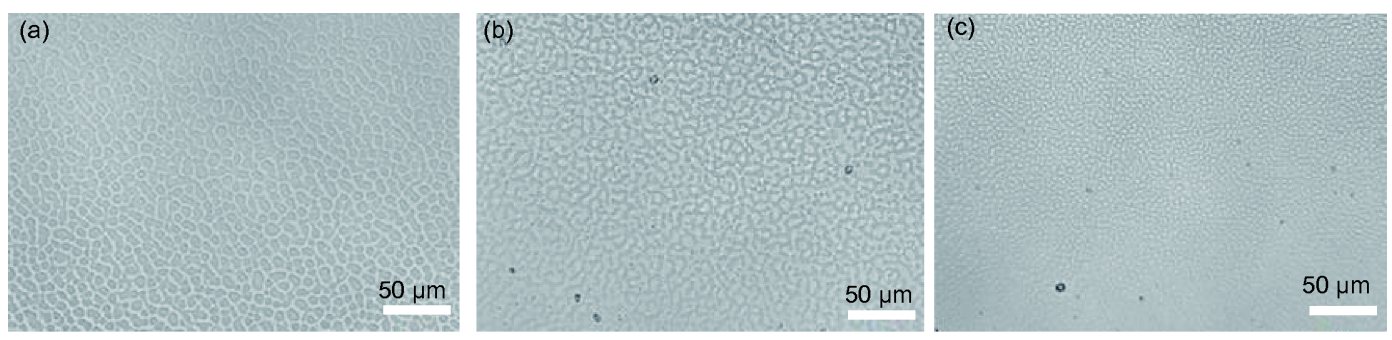

图 $7 \mathrm{PS} 60 \mathrm{PBd} 40$ 与未添加粒子(a), $10 \mathrm{wt} \%$ 的 $\mathrm{SiO}_{2} @ \mathrm{PDVB}$ Janus粒子(b), $10 \mathrm{wt} \%$ 的PBd- $\mathrm{SiO}_{2} @ \mathrm{PDVB} \mathrm{Janus}(\mathrm{c})$ 共混, 于 $150^{\circ} \mathrm{C}$ 退 火 $2 \mathrm{~h}$ 后的相形貌

Figure 7 Phase contrast optical microscopic images of PS60PBd40 blends with different particles. (a) Without particles, (b) 10 wt $\%$ of $\mathrm{SiO}_{2} @ \mathrm{PDVB}$ Janus particles, (c) $10 \mathrm{wt} \%$ of $\mathrm{PBd}-\mathrm{SiO}_{2} @ \mathrm{PDVB}$ Janus particles annealed at $150{ }^{\circ} \mathrm{C}$ for $2 \mathrm{~h}$. 

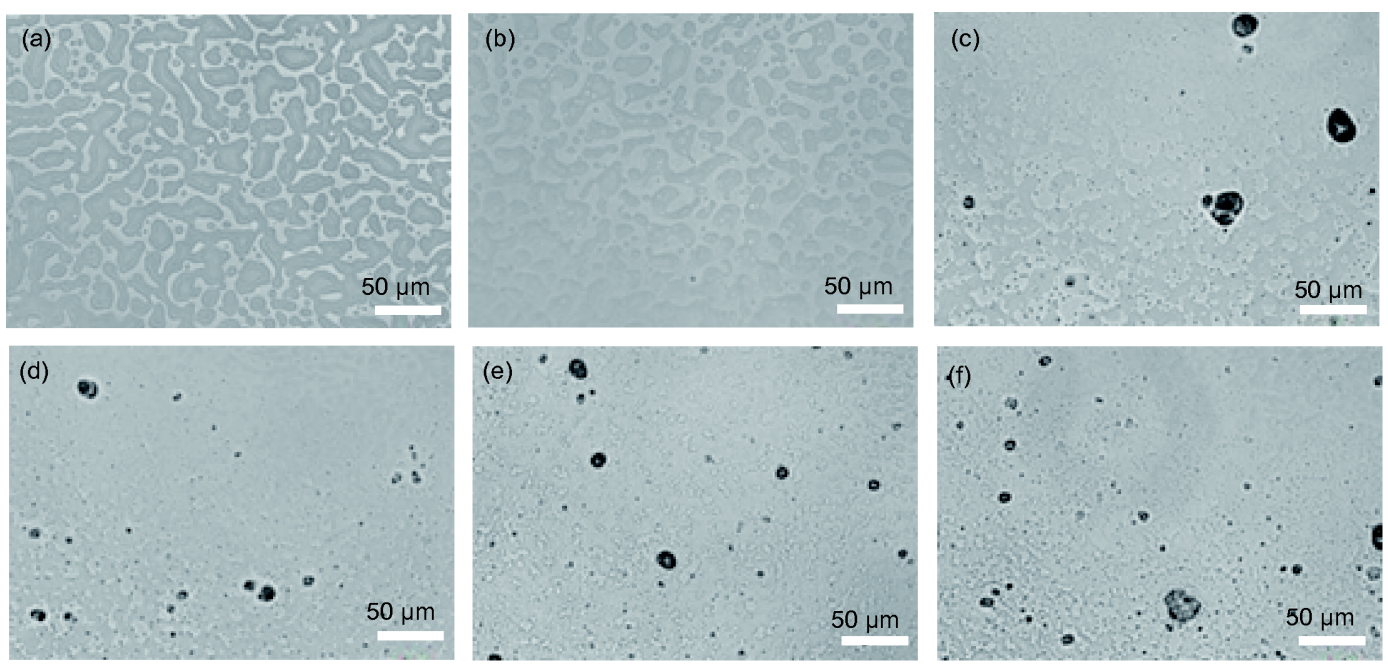

图 8 PS60PI40与 $0 \mathrm{wt} \%$ (a), $5 \mathrm{wt} \%$ (b), $10 \mathrm{wt} \%$ (c), $15 \mathrm{wt} \%$ (d) $\mathrm{PBd}-\mathrm{SiO}_{2} @ \mathrm{PDVB}$ 粒子共混后 $150^{\circ} \mathrm{C}$ 退火 $2 \mathrm{~h}$ 后的相形貌

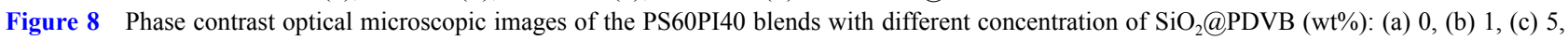
(d) 8.5 , (e) 10 , (f) 15 annealed at $150{ }^{\circ} \mathrm{C}$ for $2 \mathrm{~h}$.
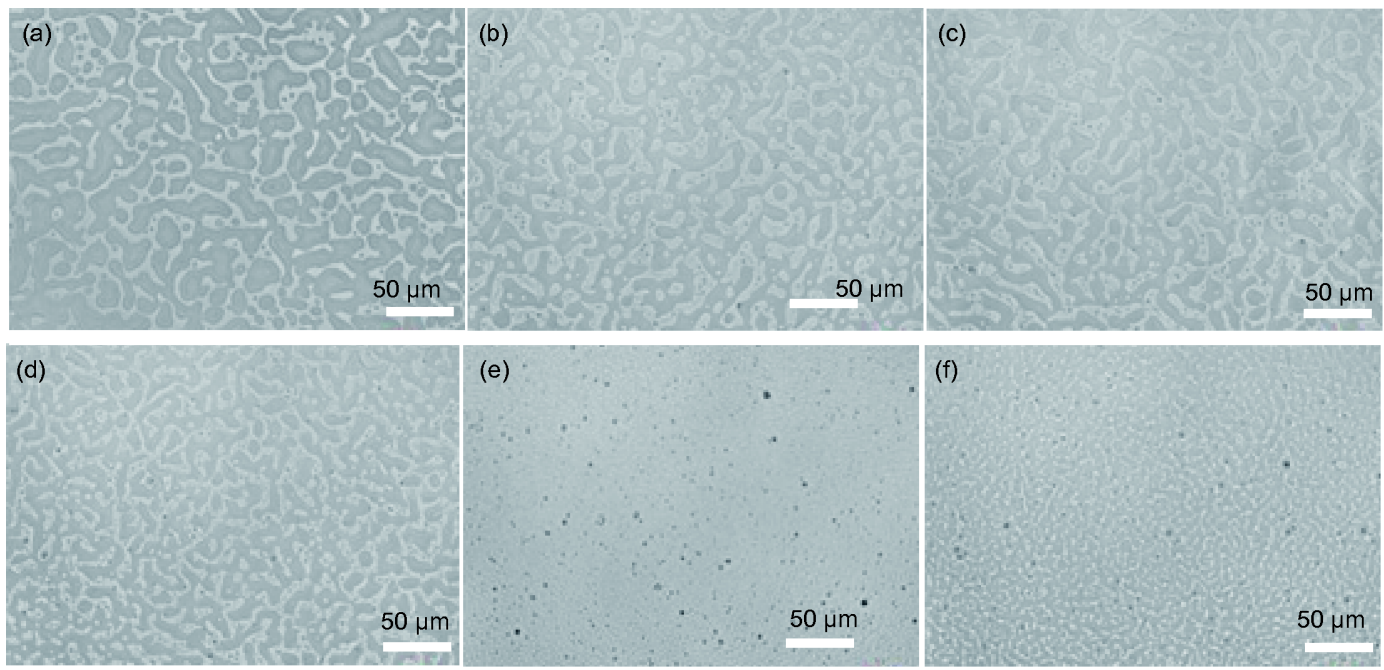

图 $9 \mathrm{PS} 60 \mathrm{PI} 40$ 与 $0 \mathrm{wt} \%$ (a), $5 \mathrm{wt} \%$ (b), $10 \mathrm{wt} \%$ (c), $15 \mathrm{wt} \%$ (d) $\mathrm{PI}_{-}-\mathrm{SiO}_{2} @ \mathrm{PDVB}$ 粒子共混,于 $150^{\circ} \mathrm{C}$ 退火 $2 \mathrm{~h}$ 后的相形貌

Figure 9 Phase contrast optical microscopic images of the PS60PI40 blends with the varied concentration of PI-SiO ${ }_{2} @ \mathrm{PDVB}$ (wt $\%$ ) : (a) 0, (b) 1, (c) 5 , (d) 8.5 , (e) 10 , (f) 15 annealed at $150{ }^{\circ} \mathrm{C}$ for $2 \mathrm{~h}$.

混物的界面张力, 并很好地平衡了 $\mathrm{SiO}_{2}$ 半球与PDVB半 球与PI和PS基底间的亲和性，从而起到了更好的增容 效果.

图10为5\%含量的 $\mathrm{SiO}_{2} @ \mathrm{PDVB}$ 与 $\mathrm{PI}-\mathrm{SiO}_{2} @ \mathrm{PDVB}$ Janus粒子在PS/PI共混物中的分布. 可以看出, $\mathrm{SiO}_{2} @$ PDVB Janus粒子团聚较明显, 且主要聚集在PS相, 改 性后的PI-SiO $\mathrm{Si}_{2} @$ PDVB Janus粒子尽管分散性较未改 性前好，但也大部分聚集在PS相，这可能是由雪人状 的 $\mathrm{SiO}_{2} @ \mathrm{PDVB}$ 粒子的结构决定的, PDVB半球对PS的
亲和性强于对PI的亲和性, 且比 $\mathrm{SiO}_{2}$ 半球的面积大, 因 此, 即便改性后可以适当减弱 $\mathrm{SiO}_{2} @ \mathrm{PDVB} J a n u s$ 粒子 对某一相(PS)的优先润湿性, 但不能使 $\mathrm{SiO}_{2}$ 半球对PI相 的亲和性完全和PDVB半球对PS的亲和性相抗衡.

\section{4 结论}

本文以雪人状的 $\mathrm{SiO}_{2} @ \mathrm{PDVB}$ Janus粒子为模板, 将阴离子聚合制备的PI及PBd分子链引入到 $\mathrm{SiO}_{2}$ 半球, 


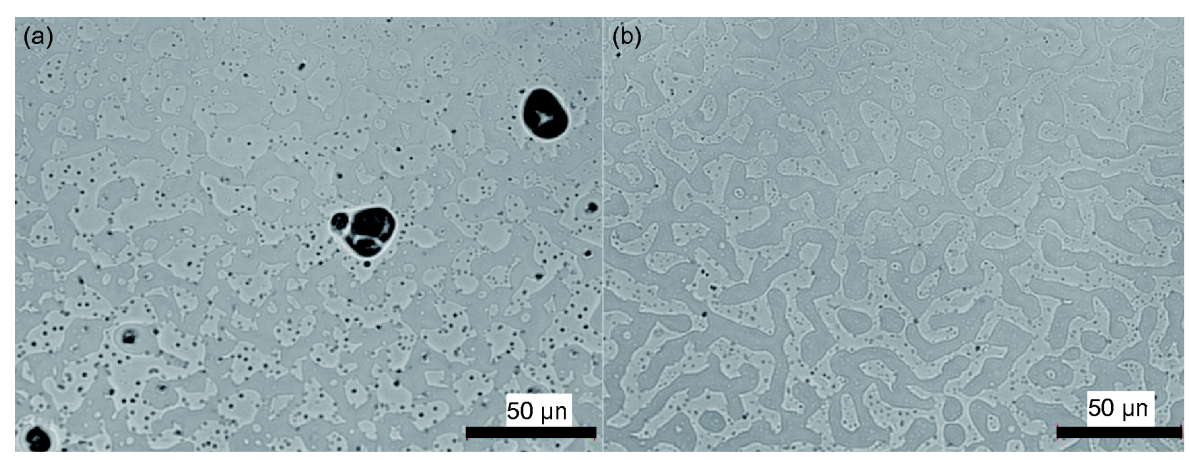

图 $10 \mathrm{PS} 60 \mathrm{PI} 40$ 与 $5 \%$ 的 $\mathrm{SiO}_{2} @ \mathrm{PDVB}(\mathrm{a}), 5 \%$ 的PI- $\mathrm{SiO}_{2} @ \mathrm{PDVB}(\mathrm{b})$ 共混,于 $150^{\circ} \mathrm{C}$ 退火 2 h后的相形貌

Figure 10 Phase contrast optical microscopic images of the PS60PI40 blends demonstrating the locationg of 5 wt $\%$ Janus particles: (a) $\mathrm{SiO}_{2} @ \mathrm{PDVB}$, (b) $\mathrm{PI}-\mathrm{SiO}_{2} @ \mathrm{PDVB}$ annealed at $150{ }^{\circ} \mathrm{C}$ for $2 \mathrm{~h}$.

制得PBd-SiO $\mathrm{Si}_{2}$ PDVBJanus粒子与PI-SiO 2 PPVB Janus粒子. 随后, 将改性前后的 $\mathrm{SiO}_{2} @ \mathrm{PDVB}$ 与 $\mathrm{PBd}-$ $\mathrm{SiO}_{2} @ \mathrm{PDVB}$ Janus粒子和PI-SiO ${ }_{2} @ \mathrm{PDVB} J a n u s$ 粒子 分别引入到PS/PBd和PS/PI的共混体系中, 通过对基体
增容作用的观察，发现引入与聚合物基底相容性好的 聚合物刷可有效提高其界面增容效果，进而调控共混 聚合物的相结构. 本研究证明, 具有双亲性的Janus粒 子有望成为下一代理想的共混聚合物增容剂.

\section{参考文献}

1 Liang F, Liu J, Zhang C, Qu X, Li J, Yang Z. Chem Commun, 2011, 47: 1231-1233

2 Walther A, Müller AHE. Chem Rev, 2013, 113: 5194-5261

3 Deng R, Liang F, Zhu J, Yang Z. Mater Chem Front, 2017, 1: 431-443

4 Chen Y, Liu Z, Qu X, Liang F, Yang Z. Chem Asian J, 2016, 11: 1785-1788

5 Yang Q, Loos K. Polym Chem, 2017, 8: 641-654

6 Liang F X,Yang Z Z. Acta Polym Sin, 2017, 6: 1-10 (in Chinese) [梁福金金, 杨振忠. 高分子学报, 2017, 6: 1-10]

7 Liu Y, Liang F, Wang Q, Qu X, Yang Z. Chem Commun, 2015, 51: 3562-3565

8 Zhao Z, Liang F, Zhang G, Ji X, Wang Q, Qu X, Song X, Yang Z. Macromolecules, 2015, 48: 3598-3603

9 Zhao B, Zhu L. Macromolecules, 2009, 42: 9369-9383

10 Liu Z, Guo R, Xu G, Huang Z, Yan LT. Nano Lett, 2014, 14: 6910-6916

11 Huang M, Li Z, Guo H. Soft Matter, 2012, 8: 6834-6845

12 Huang M, Guo H. Soft Matter, 2013, 9: 7356-7368

13 Nie H, Zhang C, Liu Y, He A. Macromolecules, 2016, 49: 2238-2244

14 Bryson KC, Löbling TI, Müller AHE, Russell TP, Hayward RC. Macromolecules, 2015, 48: 4220-4227

15 Bahrami R, Löbling TI, Gröschel AH, Schmalz H, Müller AHE, Altstädt V. ACS Nano, 2014, 8: 10048-10056

16 Wang H, Dong W, Li Y. ACS Macro Lett, 2015, 4: 1398-1403

17 Wang H, Fu Z, Dong W, Li Y, Li J. J Phys Chem B, 2016, 120: 9240-9252

18 Sun Y, Liang F, Qu X, Wang Q, Yang Z. Macromolecules, 2015, 48: 2715-2722

19 Tang C, Zhang C, Sun Y, Liang F, Wang Q, Li J, Qu X, Yang Z. Macromolecules, 2012, 46: 188-193

20 Zhao, Perrier S. Macromolecules, 2006, 39: 8603-8608

21 Mao S Z, Wang D H. Acta Polym Sin, 1990, 2: 111-114 (in Chinese) [毛诗珍, 王德华. 高分子学报, 1990, 2: 111-114]

22 Chen Q, Whitmer JK, Jiang S, Bae SC, Luijten E, Granick S. Science, 2011, 331: 199-202

23 Yan J, Bloom M, Bae SC, Luijten E, Granick S. Nature, 2012, 491: 578-581

24 Kraft DJ, Ni R, Smallenburg F, Hermes M, Yoon K, Weitz DA, van Blaaderen A, Groenewold J, Dijkstra M, Kegel WK. Proc Natl Acad Sci USA, 


\title{
Surface modification of Janus particles and their compatibilization in polymer blends
}

\author{
Xincheng Liang, Cao Zhang, Aihua He, Huarong Nie* \\ Shandong Provincial Key Laboratory of Olefin Catalysis and Polymerization, Key Laboratory of Rubber-Plastics (Ministry of Education), School of \\ Polymer Science and Engineering, Qingdao University of Science and Technology, Qingdao 266042, China \\ *Corresponding author (email: niehr@iccas.ac.cn)
}

\begin{abstract}
Janus particles with biphasic polymer brushes are promising as compatibilizers in biphasic systems due to the combination of both the particle core and the amphiphilic properties of the two compartment faces. As the traditional colloid particles, the compatibilization efficiency of Janus particles also scales with the increased amphiphilicity of particles by both phases and the enlarging of the interfacial tension aside from the increase in the radius of the particles. Meanwhile, the interfacial tension between the demixing polymer liquids is quite low, therefore a precise control over the surface chemistry of particles is absolutely necessary for the improved accomodation of particles by polymer blends and the compatibilization efficiency. In this paper, the Janus particles with rubber brushes (polybutadiene, PBd$\mathrm{SiO}_{2} @$ PDVB; polyisoprene, PI-SiO $\mathrm{S}_{2} @ \mathrm{PDVB}$ ) are synthesized by surface modification on a snowman like Janus particle $\left(\mathrm{SiO}_{2} @ \mathrm{PDVB}\right)$ template, from which PBd and PI brushes are previously synthesized by anionic polymerization, followed by their covalently attached onto $\mathrm{SiO}_{2}$ surface of JP cores. Adding such modified Janus particles into polystyrene (PS)/PI or PS/PBd blends enables offsetting to some extent the stronger interaction between PS phases and PDVB hemispheres. Resultantly, PBd-SiO $\mathrm{S}_{2}$ PDVB and PI-SiO $\mathrm{P}_{2} @ \mathrm{PDVB}$ particles character as the better efficiency in compatibilization than $\mathrm{SiO}_{2} @$ PDVB particles, which reflected as the smaller PS phase distributed in the rubber matrix. It is expected this article could contribute to the advances in the application of Janus particles in polymer blends.
\end{abstract}

Keywords: Janus particles, surface modification, compatibilization, polymer blend

doi: $10.1360 / \mathrm{N} 032018-00005$ 